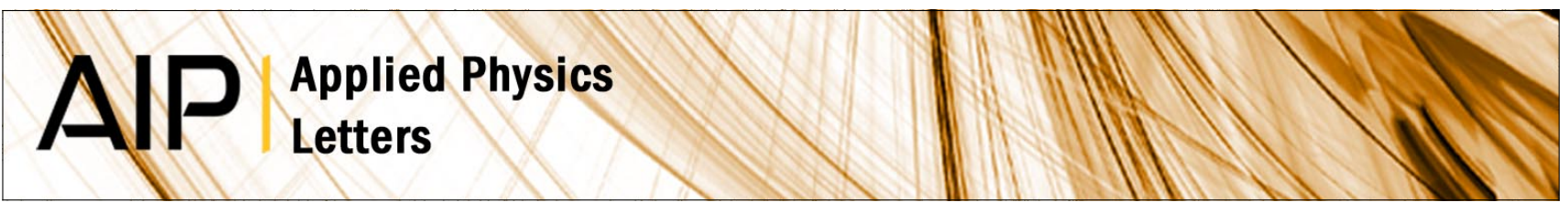

\title{
Resolving Zeeman splitting in quantum dot ensembles
}

Matthew W. Taylor, Peter Spencer, Edmund Clarke, Edmund Harbord, and Ray Murray

Citation: Appl. Phys. Lett. 102, 171909 (2013); doi: 10.1063/1.4804267

View online: http://dx.doi.org/10.1063/1.4804267

View Table of Contents: http://apl.aip.org/resource/1/APPLAB/v102/i17

Published by the American Institute of Physics.

Additional information on Appl. Phys. Lett.

Journal Homepage: http://apl.aip.org/

Journal Information: http://apl.aip.org/about/about_the_journal

Top downloads: http://apl.aip.org/features/most_downloaded

Information for Authors: http://apl.aip.org/authors

\section{ADVERTISEMENT}

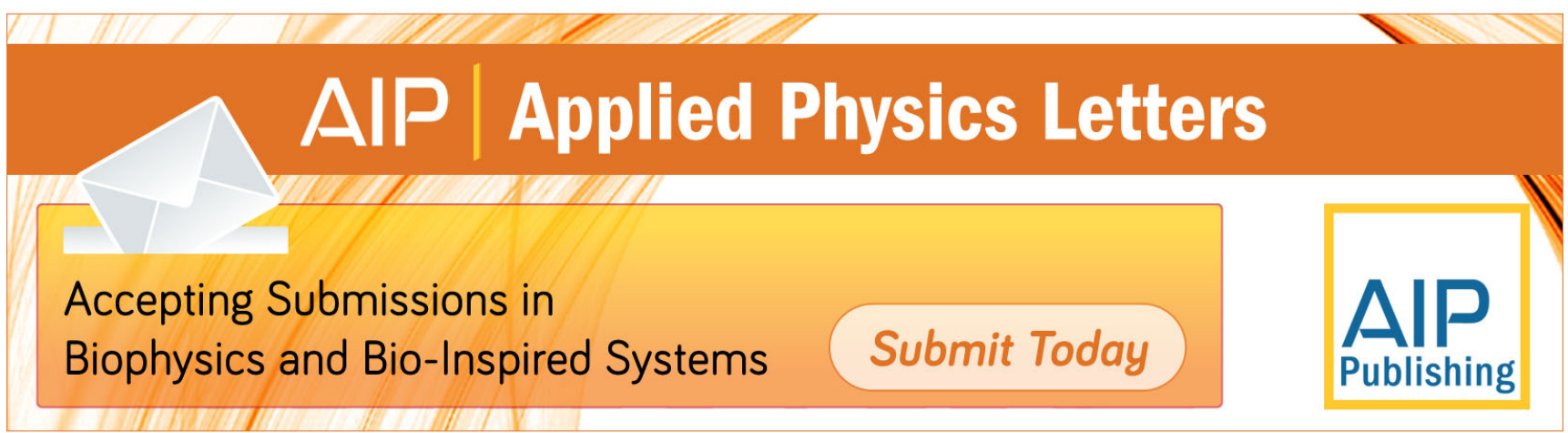




\title{
Resolving Zeeman splitting in quantum dot ensembles
}

\author{
Matthew W. Taylor, ${ }^{1, a)}$ Peter Spencer, ${ }^{1}$ Edmund Clarke, ${ }^{2}$ Edmund Harbord, ${ }^{3}$ \\ and Ray Murray ${ }^{1}$ \\ ${ }^{1}$ Department of Physics, The Blackett Laboratory, Imperial College London, Prince Consort Road, \\ London SW7 2AZ, United Kingdom \\ ${ }^{2}$ EPSRC National Centre for III-V Technologies, University of Sheffield, Sheffield S1 3JD, United Kingdom \\ ${ }^{3}$ NanoQUINE, The University of Tokyo, 4-6-1, Komaba, Meguro-ku, Tokyo 153-8505, Japan
}

(Received 15 March 2013; accepted 23 April 2013; published online 3 May 2013)

\begin{abstract}
This letter presents a technique for the investigation of the fine structure and spin properties of quantum dot (QD) ensembles, allowing measurement of QD parameters previously accessible only from studies of individual QDs. We show how $\sim \mu \mathrm{eV}$ splittings can be deduced from information contained in the shape of the ensemble polarization spectra and demonstrate the effectiveness of this technique by measuring Zeeman splittings, $g$-factors, and sensitivity to QD fine structure effects. (C) 2013 AIP Publishing LLC. [http://dx.doi.org/10.1063/1.4804267]
\end{abstract}

There is continuing interest in spin and polarization effects in self-assembled quantum dots (QDs) for applications in quantum information, quantum communication, and spintronics. Despite extensive effort there is no consensus on important properties such as $g$-factors, fine structure, and spin splittings. Much of the existing work has concentrated on the determination of these QD properties using advanced microscopy (e.g., Refs. 1-4). Such experiments are conceptually simple, allowing precise measurement of individual QD emission lines, but are limited by the stringent experimental requirements for resolving emission from single QDs. By contrast, optical measurements obtained from QD ensembles avoid the stability and detection problems associated with single dot measurements. The use of ensembles also provides rapid access to a statistical average of QD properties sampled over a large number of dots, thus negating the requirement to make repeated measurements on individual QDs. However, inhomogeneous broadening of the QD ensembles (several tens of meV) means that it is not possible to resolve emission lines from individual QDs. This work presents a technique capable of measuring energy splittings between differently polarized emission lines, corresponding to the recombination of excitons in individual QDs, across a whole QD ensemble. We demonstrate the simplicity of the technique by applying it to a study of Zeeman splitting in QD ensembles and show that the procedure allows access to measurements of important structural and spin parameters formerly only directly accessible using single QD microscopy.

The QD ensemble samples were grown by molecular beam epitaxy on semi-insulating GaAs (001) substrates. A single InAs QD layer, with typical areal QD density $2 \times 10^{10} \mathrm{~cm}^{-2}$, was capped with GaAs and sandwiched between AlGaAs barriers, to aid carrier confinement. The low growth rate $(0.014$ monolayers per second) results in large, indium rich dots which emit at $1170 \mathrm{~nm}$ at $10 \mathrm{~K}$, with a narrow Gaussian-like ensemble full width at half maximum (FWHM) of $\sim 30 \mathrm{meV}$. For this work undoped and $p$-doped (at a doping level of 3 holes/QD) samples were chosen to

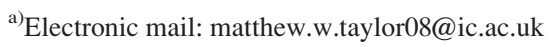

highlight the applicability of the technique. Further structure and growth details of the ensembles used may be found in Harbord et al. ${ }^{5}$ All experiments were performed with the samples maintained at $8 \mathrm{~K}$ in a magneto-cryostat, capable of applying up to $5 \mathrm{~T}$ to the QD sample in the Faraday geometry. Unpolarized optical excitation was provided by a Helium-Neon laser at an excitation wavelength of $632.8 \mathrm{~nm}$. The full polarization state of the emitted light across the ensemble was resolved using a Stokes polarimeter employing a rotatable quarter wave plate and a fixed linear polarizer. A low excitation level was used throughout, sufficient to suppress emission from excited states, which can significantly alter the QD ensemble photoluminescence (PL) polarization spectrum. 6,7

QDs subjected to low power, unpolarized excitation, and a magnetic field in the growth direction will exhibit polarized exciton emission lines split by an energy $\Delta_{Z}$ due to the Zeeman effect. These lines are distinguished by their opposing circular polarization (CP), resulting from their spin orientation. The Zeeman Hamiltonian in an external magnetic field $B_{z}$ is given by ${ }^{2}$

$$
\mathcal{H}_{\text {Zeeman }}^{F}\left(B_{z}\right)=-\mu_{B}\left(g_{e, z} S_{e, z}-\frac{g_{h, z}}{3} J_{h, z}\right) B_{z},
$$

where $\mu_{B}$ is the Bohr magneton, $g_{e, z}$ and $g_{h, z}$ are the electron and heavy-hole $g$-factors in the Faraday geometry, and $S_{e, z}= \pm 1 / 2$ and $J_{h, z}= \pm 3 / 2$ their spin projections. The resulting Zeeman splitting of the counter-polarized bright excitons, with angular momentum $S_{e, z}+J_{h, z}= \pm 1$, will therefore be $\Delta_{Z}=g_{e x, z} \mu_{B} B_{z}$, where $g_{e x, z}$ is the bright exciton $g$-factor $\left(g_{e, z}+g_{h, z}\right)$. A typical PL experiment made on a QD ensemble will sample many millions of such QDs, due to the high areal density and finite excitation spot size. This results in an inhomogenously broadened emission spectrum such as seen in Figure 1, obtained from an undoped sample. For an ensemble of QDs, with an average $g$-factor $g_{\text {ex } z}^{*}$, a Zeeman splitting will be induced between the counter-polarized emission components of every QD. ${ }^{1}$ Figure 1 also shows the measured degree of circular polarization $\rho_{I}$ given by $\rho_{I}=\left(I_{\sigma^{+}}-I_{\sigma^{-}}\right) /\left(I_{\sigma^{+}}+I_{\sigma^{-}}\right)$, of the sample in an external magnetic field, where $I_{\sigma^{+}}\left(I_{\sigma^{-}}\right)$is the PL intensity of the 
$\sigma^{+}\left(\sigma^{-}\right)$circularly polarized emission. The polarization spectrum varies across the ensemble, inverts when the magnetic field is reversed, and is negligibly small when the field is removed. In all three cases there is no discernible change in the shape of PL emission intensity spectrum.

Using the intensity and polarization information provided by the polarimeter, the PL emission can be split into its $\sigma^{+}$(black) and $\sigma^{-}$(red) components as shown in Figure 2(a) for an applied field of $5 \mathrm{~T}$. This reveals that the application of the field results in a small $(\sim 0.5 \mathrm{meV})$ energy splitting between the polarization peaks, with the $\sigma^{+}$emission component at a lower energy than $\sigma^{-}$. A difference in amplitude between the components is also observed, corresponding to a net polarization. To investigate how the polarization spectrum may be produced by the Zeeman splitting of the QD states we analyze the factors which contribute to the shape of the polarization spectrum.

The emission spectrum of a QD ensemble is typically approximated using a Gaussian form

$$
G(E)=A \mathrm{e}^{-\left[\frac{\left(E-E_{c}\right)}{\Gamma}\right]^{2}},
$$

where $A$ is the amplitude, $E_{c}$ the peak energy, and the FWHM is $2 \sqrt{\ln 2} \times \Gamma$. Such a fit is shown in Figure 2(c) and can be seen to reproduce the form of the PL emission from the peak to the lower energy side of the spectrum. The real QD ensemble deviates from true Gaussian form on the high energy side of the PL peak. This is attributed to the nonuniform distribution of QD sizes, ${ }^{7,8}$ in particular, the presence of sub-populations of smaller dots. ${ }^{7,9}$ Nevertheless, the low energy Gaussian-like region observed is typical of QD ensembles, allowing such a treatment to be applied to a wide range of samples. The QD excited states, which become apparent at higher laser power, ${ }^{7}$ do not contribute significantly to this spectrum due to the low level excitation used. If a small energy splitting $\Delta$ exists between the differently circularly polarized emission lines of every individual QD, the ensemble emission may be approximated as two counter polarized Gaussian distributions: $G_{\sigma+}$ and $G_{\sigma-}$ separated by

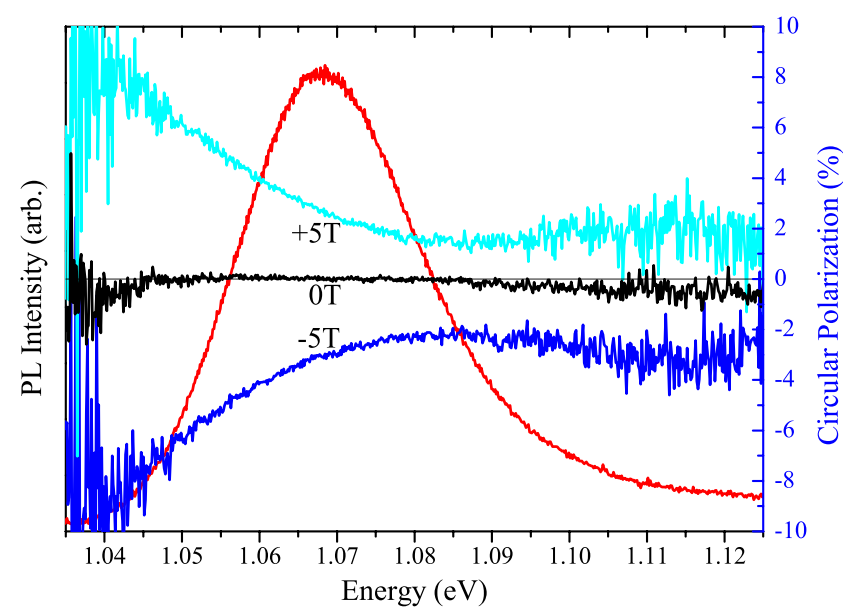

FIG. 1. PL spectrum (red) and circular polarization obtained from an undoped QD ensemble, using unpolarized excitation under applied magnetic fields of $-5 \mathrm{~T}$ (blue), $0 \mathrm{~T}$ (black), and $+5 \mathrm{~T}$ (cyan) along the growth direction.

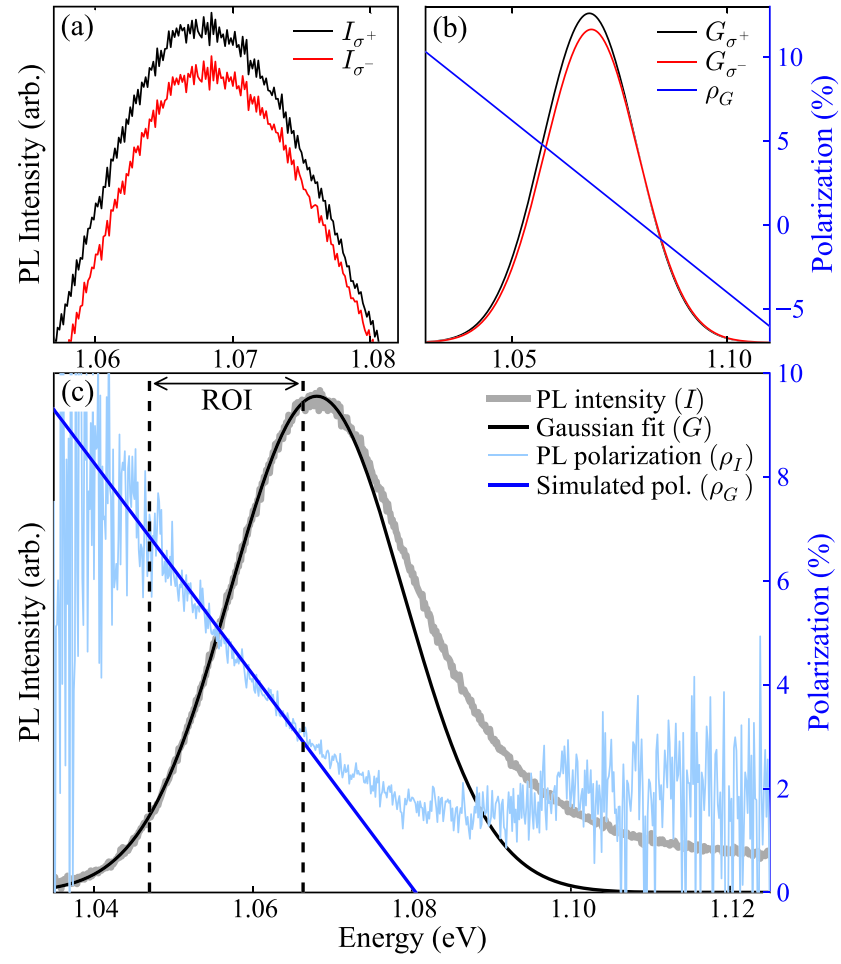

FIG. 2. (a) Emission peak at $5 \mathrm{~T}$ resolved into $\sigma^{+}$(black) and $\sigma^{-}$(red) components: a small overall polarization $\left(\rho_{A}\right)$ and splitting $(\Delta)$ is evident between the two. (b) Polarization $\left(\rho_{G}\right.$, blue) produced by a pair of split Gaussian distributions $\left(\Delta=0.49 \mathrm{meV}, \rho_{A}=2.5 \%\right)$. (c) Ensemble PL emission at $5 \mathrm{~T}$ (gray) approximated by a Gaussian distribution (black). The PL polarization $\left(\rho_{I}\right.$, light blue) is compared with the linear form resulting from two split Gaussians $\left(\rho_{G}\right.$, blue) and is seen to match over the region where the ensemble follows the Gaussian shape (ROI).

an energy corresponding to the ensemble average splitting. There may also be an overall net circular polarization $\rho_{A}=\left(A_{\sigma^{+}}-A_{\sigma^{-}}\right) /\left(A_{\sigma^{+}}+A_{\sigma^{-}}\right)$arising from an imbalance of spin population. Such a situation is simulated in Figure 2(b), where the black and red curves represent the $\sigma^{+}$ and $\sigma^{-}$emission, with an energy splitting $\Delta=0.49 \mathrm{meV}$ and polarization $\rho_{A}=2.5 \%$ between the two. This corresponds to a shifting to lower energy of the $\sigma^{+}$Gaussian with a corresponding increase in its amplitude, which may be expected as a result of spin redistribution into the lower energy level at low temperature. ${ }^{10}$ The resulting emission polarization spectrum $\left(\rho_{G}\right)$ is shown as the blue line and displays a linear form. This polarization $\rho\left(E^{\prime}\right)=\left(G_{\sigma+}-G_{\sigma-}\right) /\left(G_{\sigma+}+G_{\sigma-}\right)$, where $E^{\prime}=E-E_{0}$ and $E_{0}$ is the midpoint between the peaks, is given by

$$
\rho\left(E^{\prime}\right)=\frac{A \mathrm{e}^{\frac{-\left(E^{\prime}-\Delta / 2\right)^{2}}{\Gamma^{2}}}-\mathrm{e}^{\frac{-\left(E^{\prime}+\Delta / 2\right)^{2}}{\Gamma^{2}}}}{A \mathrm{e}^{\frac{-\left(E^{\prime}-\Delta / / 2\right)^{2}}{\Gamma^{2}}}+\mathrm{e}^{\frac{-\left(E^{\prime}+\Delta / 2\right)^{2}}{\Gamma^{2}}}},
$$

where $A=A_{\sigma+} / A_{\sigma_{-}}$, which reduces to

$$
\rho\left(E^{\prime}\right)=\tanh \left(\frac{E^{\prime} \Delta}{\Gamma^{2}}+\frac{\ln A}{2}\right)=\lim _{E^{\prime} \Delta \rightarrow 0} \frac{E^{\prime} \Delta}{\Gamma^{2}}+\rho_{A} .
$$

Thus, a QD ensemble with a Gaussian emission profile and an energy splitting between spin states will exhibit a linear slope $\Delta / \Gamma^{2}$ in its polarization spectrum, offset by the overall spin polarization $\rho_{A}$. Figure 2(c) shows that the 
behavior observed for the real QD ensemble closely matches that derived in Eq. (4) over the region of interest (ROI) where the ensemble displays a Gaussian shape. Thus $\Delta$ and $\rho_{A}$ can be easily obtained from a linear fit to the polarization spectrum over this region, yielding a simple method for measuring spin splittings and polarisation magnitude in QD ensembles. Since the gradient of the polarisation is sensitive only to the relative energy splitting of the spin states, other magnetic field effects that are not spin dependent and therefore only affect the PL peak energy, such as the diamagnetic energy shift, ${ }^{2}$ are automatically separated. This allows the spin splitting component to be easily isolated without complication from other effects. The linear form across the ensemble means that even a very small change in splitting can result in a significant change in polarization slope, thus yielding a very high precision to the measurement. Due to the $\Gamma^{2}$ factor in the polarization gradient the technique favors QD ensembles with a narrow, well defined Gaussian FWHM. The deviation from the linear gradient outside the indicated ROI results from the deviation of the emission from an ideal Gaussian profile. A similar analysis of the shape of these regions could be used to further model the behavior of the polarization. However, confining the analysis to the linear region yields the simplest approach and can be applied to a wide range of QD samples.

This technique can now be used to resolve and quantify Zeeman splittings for an ensemble of QDs. Using Eq. (4) the value of $\Delta_{Z}$ was measured over the available magnetic field range for the undoped and $p$-doped samples, and this is shown in Figure 3. For magnetic fields above $1 \mathrm{~T}$, where Zeeman effects dominate, ${ }^{1,2}$ a linear increase in $\Delta_{Z}$ is observed with increasing field magnitude for both samples. This allows an ensemble average $g$-factor $g_{e x, z}^{*}=-1.92 \pm 0.04$ to be determined for the undoped sample and $g_{e x, z}^{*}=-1.91 \pm 0.02$ for the sample doped at a level

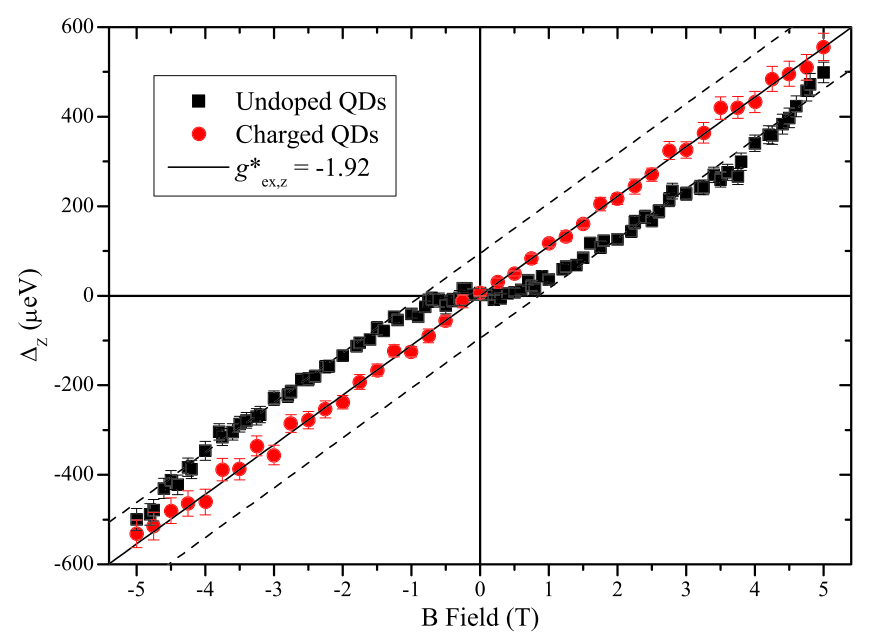

FIG. 3. Variation of measured Zeeman splitting $\left(\Delta_{Z}\right)$ with applied magnetic field as deduced from the linear slope in the PL CP polarization spectrum. Black squares show the $\mathrm{CP}$ splitting for an undoped QD ensemble and red circles for a structurally similar ensemble doped at a level of 3 holes/QD. The ensemble average $g$-factor $\left(g^{*}\right)$ can thus be derived from the linear slope in $\Delta_{Z}$ (black lines show a slope corresponding $g^{*}=1.92$ ). The difference between the ensembles can be attributed to the QD fine structure splitting, which dominates over the Zeeman behavior at lower field values but may be eradicated via the addition of resident carriers. of 3 holes/QD. These values compare favorably with experiments performed on single QDs emitting at similar energies $^{3,11,12}$ and provide confidence in the applicability of the technique.

For the undoped sample, at magnetic field values $|\mathrm{B}|<1 \mathrm{~T}$, the behavior diverges from the linear Zeeman form. We attribute this to the effects of QD asymmetry and the electron-hole exchange interaction. ${ }^{1,2}$ The exchange interaction mixes the exciton states into linearly polarized (LP) states split by the fine structure splitting (FSS). An interplay between the LP FSS and CP Zeeman splitting suppresses the increase in $\Delta_{Z}$ with applied field until the Zeeman splitting is large compared to the exchange splitting, at which point the linear form returns. ${ }^{2}$ By charging the QDs the effects of the fine-structure splitting can be removed via cancellation of the exchange interaction, ${ }^{2}$ and this is evident in the sample doped at 3 holes/QD (chosen such that only a negligible number of QDs may be expected to be uncharged). Here a linear increase in splitting between CP emission lines is observed over the whole field range. Interestingly, at high magnetic fields, a deviation from the linear form is also observed in the undoped sample. Such behavior may result from coupling between bright and dark exciton states in the QDs, ${ }^{2}$ which is suppressed in the doped QD sample due to the prevalence of charged exciton states. The sensitivity to the QD fine structure could be used to optimise growth of QD samples for future photonic applications, where minimisation of the FSS is important and demonstrates that this technique allows investigation of a range of properties using QD ensembles. The technique may also be extended to consider direct resolution of the QD fine structure via measurement in the linear polarisation basis or spin dependent properties induced through circularly polarised excitation and such investigations may form the basis of future publications.

In summary we present a technique to investigate the electronic structure and spin properties of semiconductor QDs. This method uses previously neglected information contained in the polarization spectrum of a QD ensemble to determine the energy splitting of individual QD lines. We have validated the technique by measuring Zeeman splittings, which are much smaller than the ensemble FWHM of $30 \mathrm{meV}$, and determined average $g$-factors for both undoped and $p$-doped QD samples. Comparison of these samples at low magnetic fields suggests that the technique is also sensitive to the QD fine structure splitting, thus showing that the method may be used as a general technique to probe multiple aspects of QD electronic structure. This provides an alternative to single dot measurements: the method is experimentally simpler, is more representative of a typical dot, and is not confined to shorter wavelengths. It also avoids the necessity for low density, post-growth processed samples, providing a general tool for the wafer-level investigation and growth optimization of QDs for future photonic applications.

${ }^{1}$ M. Bayer, A. Kuther, A. Forchel, A. Gorbunov, V. B. Timofeev, F. Schäfer, J. P. Reithmaier, T. L. Reinecke, and S. N. Walck, Phys. Rev. Lett. 82, 1748-1751 (1999).

${ }^{2}$ M. Bayer, G. Ortner, O. Stern, A. Kuther, A. A. Gorbunov, A. Forchel, P. Hawrylak, S. Fafard, K. Hinzer, T. L. Reinecke, S. N. Walck, J. P. Reithmaier, F. Klopf, and F. Schäfer, Phys. Rev. B 65, 195315 (2002). 
${ }^{3}$ T. Nakaoka, T. Saito, J. Tatebayashi, S. Hirose, T. Usuki, N. Yokoyama, and Y. Arakawa, Phys. Rev. B 71, 205301 (2005).

${ }^{4}$ A. I. Tartakovskii, T. Wright, A. Russell, V. I. Fal'ko, A. B. Van'kov, J. Skiba-Szymanska, I. Drouzas, R. S. Kolodka, M. S. Skolnick, P. W. Fry, A. Tahraoui, H.-Y. Liu, and M. Hopkinson, Phys. Rev. Lett. 98, 026806 (2007). ${ }^{5}$ E. Harbord, P. Spencer, E. Clarke, and R. Murray, Phys. Rev. B 80, 195312 (2009).

${ }^{6}$ G. Kioseoglou, M. Yasar, C. Li, M. Korkusinski, M. Diaz-Avila, A. Hanbicki, P. Hawrylak, A. Petrou, and B. Jonker, Phys. Rev. Lett. 101, 227203 (2008).

${ }^{7}$ M. W. Taylor, E. Harbord, P. Spencer, E. Clarke, G. Slavcheva, and R. Murray, Appl. Phys. Lett. 97, 171907 (2010).
${ }^{8}$ E. Harbord, P. Spencer, E. Clarke, and R. Murray, J. Appl. Phys. 105, 033507 (2009).

${ }^{9}$ S. J. Lee, S. K. Noh, J. W. Choe, and E. K. Kim, J. Cryst. Growth 267, 405 (2004).

${ }^{10}$ Such spin redistribution effects may be expected to reduce at higher temperature. However, the measurement of the underlying energy splitting outlined in this paper, being purely spectroscopic, is not be expected to display a significant temperature dependence.

${ }^{11}$ T. Nakaoka, T. Saito, J. Tatebayashi, and Y. Arakawa, Phys. Rev. B 70, 235337 (2004).

${ }^{12}$ N. Cade, H. Gotoh, H. Kamada, H. Nakano, and H. Okamoto, Phys. Rev. B 73, 115322 (2006). 